\title{
Polarization Distributions in PVDF Obtained by Poling under Constant Current Condition
}

\author{
G. Neumann, E. Bihler, G. Eberle, W. Eisenmenger \\ 1. Physikalisches Institut, Universität Stuttgart, \\ Stuttgart, Germany
}

\begin{abstract}
Since the resistivity of PVDF films increases when the films are poled, the voltage across the samples must be increased during the poling process in order to keep the current constant. Under these conditions we observe the formation of a polarization zone in the center of the film depth. The thickness of the polarization free zone close to the film surfaces is found to decrease with larger current densities. The results are consistent with a model assuming charge trapping in deep traps associated with the orientation of polar crystallites.
\end{abstract}

\section{Introduction}

The poling of PVDF films in high electric fields of $\mathrm{MV} / \mathrm{cm}$ leads to a strong electric polarization. Part of this polarization remains permanent after removing the poling field. This remanent polarization is responsible for the piezoelectricity found in poled PVDF films.

In most cases poling is done by applying a constant field to the films. The time development of the polarization and its spatial distribution across the film thickness can be measured using pressure wave techniques $[3,1]$. With lower field strength $(<1 \mathrm{MV} / \mathrm{cm})$ spatially inhornogeneous polarization distributions are observed. This gives evidence for the influence of space charges on the formation of the remanent polarization [4].

Poling the films under the condition of constant charging allows to compare the experimental results with a theoretical model. Together with the independent measurement of the dielectric displacement with the PPS (piezoelectrically generated pressure step) technique it is possible to seperate the displacement current from the true charge transport through the films.

\section{Experimental}

Figure 1 shows the block diagram used for poling the samples.

During the poling process the time development of the spatial charge and polarization distribution was measured using the pressure step technique (PPS $[3,1])$. When using the PPS technique the rear site of the sample has to be at ground potential. Therefore the current has to be measured at high potential. 
Figure 1: Block diagram of the circuit used for poling the samples. The current

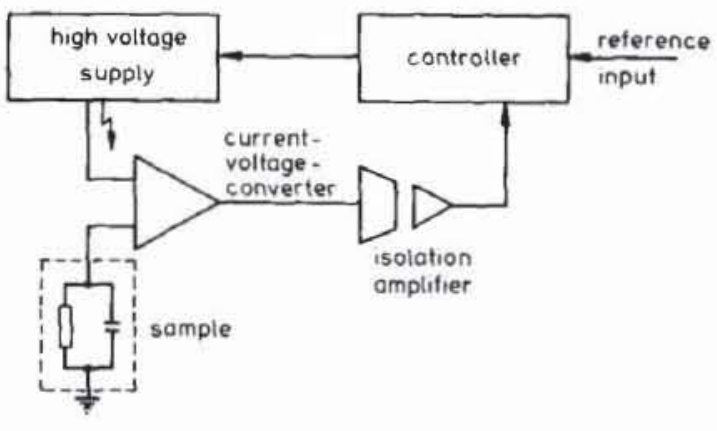

through the samples is converted into a voltage by an operational amplifier and fed through an isolation amplifier into an controller of the high voltage supply. During the poling procedure the dielectric displacement in the sample is monitored with the pressure step technique. (The sample is symbolized by a capacitor with a leakage resistor in parallel in the circuit diagram.)

We developed a ground-isolated ampere meter to measure the current $j_{0}$ into the sample. A controller for the high voltage supply keeps the measured current through the sample constant (see Figure 1).

We used commercial samples supplied from Solvay, Belgium. The films had a thickness of $38 \mu \mathrm{m}$. Almost all crystallites in this material are of the polar $\beta$-phase.

\section{Results}

During the poling process with constant current density the voltage across the sample increases. Figure 2 shows this behavior for the example of $5 \mathrm{nA} / \mathrm{cm}^{2}$ current density.

The corresponding development of the polarization is shown in Figure 3.

At the beginning we observe that the polarization is homogeneously distributed over the film thickness. But with increasing time we observe charge injection at both electrodes. The injected space charges reduce the field at the electrodes and increase the field in the center of the film. In this central zone the dipoles are oriented to a higher degree, thus forming a polarization layer in the film center.

Together with the development of this central polarization zone the resistance of the films increases. Therefore the voltage has to be increased in order to keep the current constant. The space charge distribution defines where the polarization develops.

After approx. 50000 seconds $(\approx 14$ hours) a time stationary state is approached. In this state the applied potential becomes constant and the polarization distribution does not change any more.

With higher current densities the potential increases faster and the thickness of the central polarization zone is found to be larger. We could not observe 


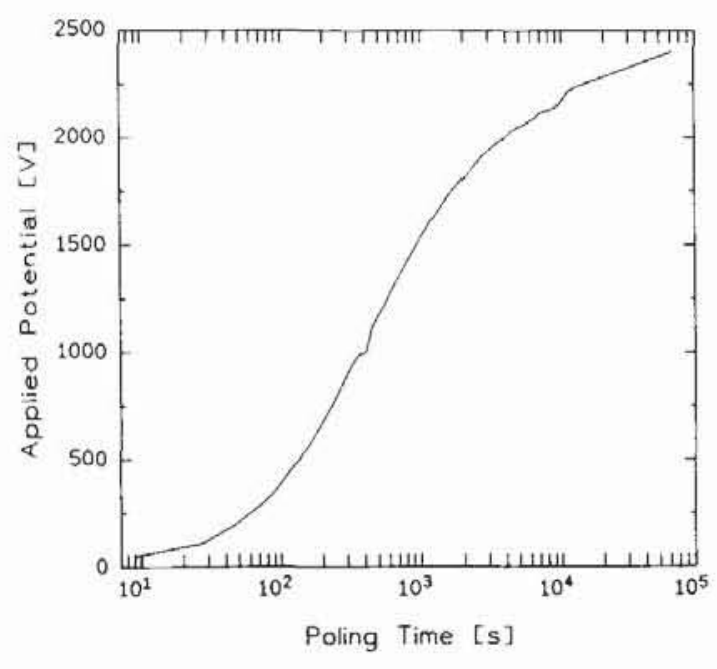

Figure 2: Increase of the applied external potential during poling with a constant current of 5 $\mathrm{nA} / \mathrm{cm}^{2}$.

the time stationary state for current densities larger than $10 \mathrm{nA} / \mathrm{cm}^{2}$ because the potential exceeded the maximum isolation voltage of the current/voltage converter (compare Figure 1) before reaching the stationary state.

Figure 5 shows the polarization distributions obtained with larger current densities. The polarization zones develop thicker with larger current densities.

\section{Discussion}

The simplest model is to assume that the poling process consists basically of the charging of a capacitor. In the case of constant current condition during the poling the time integrated current density gives the charge density on the capacitor surface. This charge density is equivalent to the dielectric displacement measured with the pressure step technique.

Figure 4 shows the time dependence of the maximum value of the displacement measured during poling with the pressure step technique. The same data as in Figure 3 is used. The integrated current density of $5 \mathrm{nA} / \mathrm{cm}^{2}$ gives a straight line marked " $a$ " in Figure 4 .

The experimental data points fall below this line, indicating a finite parallel conductivity of the sample. Part of the current flows through the sample. Correspondingly the dielectric displacement falls below the straight line.

If the sample is regarded as a capacitance with a leakage resistor in parallel, the time dependence of the dielectric displacement will follow an exponential law with the time constant $\tau=\epsilon_{0} \epsilon \rho$. For PVDF $\tau$ is of the order of 100 to 1000 $\mathrm{s}$. The corresponding curve is shown in Figure 4 marked "b". Since saturation is reached after more than $50000 \mathrm{~s}$ in the experiment this curve does not give the correct time dependence.

For a model which gives more account to the specific behavior of PVDF a nonlinear response of the polarization with applied electric field must be included. The dielectric displacement $D$ can be expressed by a Taylor series 


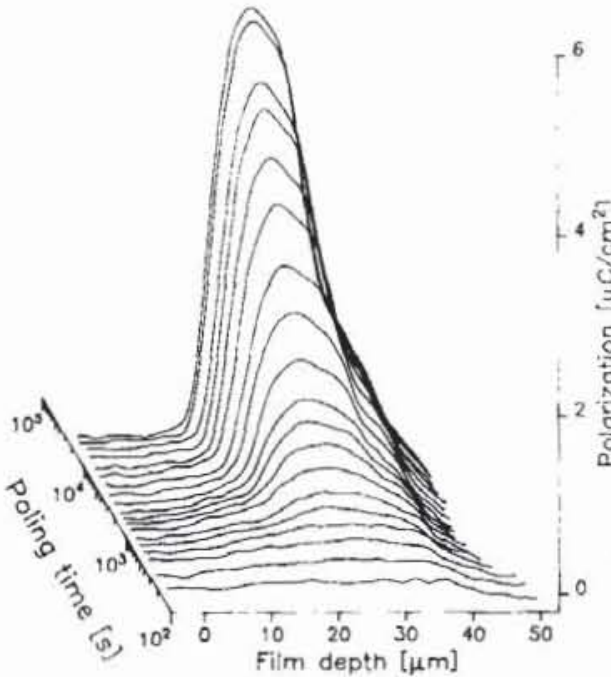

Figure 3: Time development of the polarization distribution in PVDF films during poling with a constant current density of $5 \mathrm{nA} / \mathrm{cm}^{2}$.

depending on the electric field $E$. The coefficients are the corresponding dielectric permittivities $\epsilon_{i}$.

$$
D=\epsilon_{0}\left(\epsilon_{1} E+\epsilon_{2} E^{2}+\ldots\right)
$$

For simplicity we will assume that the polarization due to the orientation of dipoles in PVDF contribute only to the quadratic term $\epsilon_{2}$ and $\epsilon_{1}$ contains the contribution of electronic and ionic polarizability. Since the electronic and ionic contributions $\left(\epsilon_{1}=3.5\right)$ are much smaller than the contribution of oriented dipoles $\left(\epsilon \approx 100\right.$ at high fields), we will neglect $\epsilon_{1}$ and use only the quadratic term.

The increase of the sample resistance with the poling time (see Figure 2), which coincides with the development of the polarization zone can be explained by a model, describing the formation of traps connected with the orientation of crystallite dipoles. These traps are located at the boundary between crystalline and amorphous regions, where the oriented dipoles of a crystallite end.

The charge carriers, which approach the boundary of the polarization zone are trapped and the conductivity through the polarization zone is therefore reduced. We take this into account by assuming the specific resistance $\rho$ to be a linear function of the electric field:

$$
\rho=\rho_{0}+b E
$$

We can estimate the values for the coefficients in 1 and 2 from the measured data. The basic resistance $\rho_{0}$ is calculated from the measured potential at the beginning, when the field is not large enough to orient the crystallite dipoles. The value for $b$ can be calculated from the long time stationary value of the applied potential at the end of the poling time. 


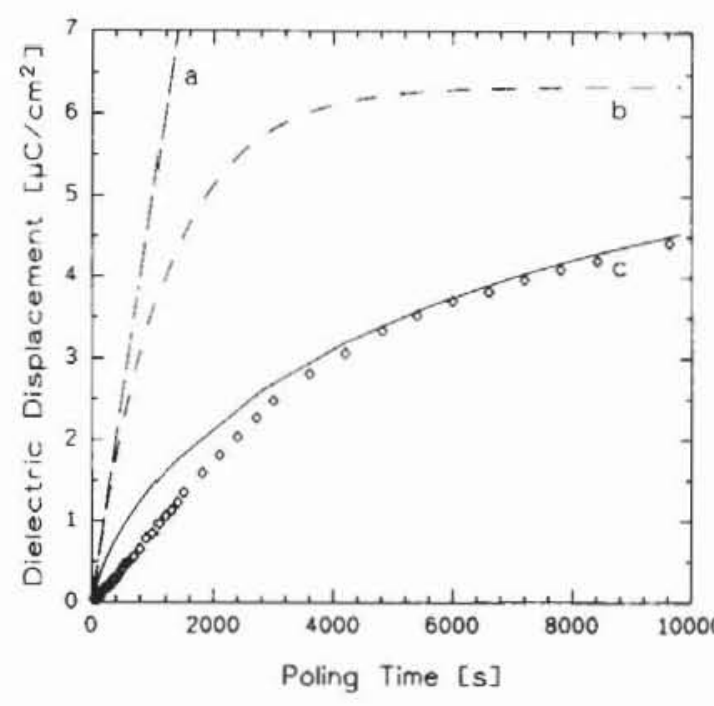

Figure 4: Time dependence of the maximum dielectric displacement taken from the polarization distribution in Figure 1 (single data points) compared with theoretical models. Line marked " $a$ ": If the sample conductivity is neglected the dielectric displacement equals the integrated current density. Line marked " $b$ ": Charging of a capacitor with a leakage resistor in parallel. $\left(\tau=\epsilon_{0} \epsilon \rho=\right.$ $1200 s ; \epsilon=100 ; \rho=$ $10^{14} \Omega \mathrm{cm}$ ) Line marked " $\mathrm{c}$ ": Improved model with electric field dependent polarization and conductivity.

The total current $j_{0}$, which is kept constant during the experiments consists of two parts: the displacement current and the charge flow.

$$
j_{0}=\dot{D}+\rho^{-1} E=\text { const } .
$$

This differential equation for $D$ can be integrated using numerical methods (Runge-Kutta [5]). The calculated time development of the displacement with the above estimated coefficients gives the line marked "c" in Figure 4.

This model can represent the measured data with good agreement. It does not describe the spatial distribution of charges and polarization along the thickness direction, since only the time dependence of the variables $D$ is used in 3 .

A complete description of space and time derivates can be obtained using the Maxwell-Equations together with 3. The resulting partial differential equation can be integrated in space for the time stationary case ( all time derivatives vanish ) if the current $j_{0}$ is kept constant [4].

The analytical solution gives an internal polarization zone:

$$
P(x)=\sqrt{j_{0} c\left(\exp \frac{x}{\delta}-1\right)}
$$

$\delta$ and $c$ include parameters concerning the model (density of traps with applied field) and the material (linear polarizability due to the polar crystallites, mobility of the charge carriers). The solution diverges for $x \rightarrow \infty$ since no saturation of the polarization is taken into account. Only one polarity of homo charges is considered. Therefore the solution applies only for one side of the films. This solution is compared in Figure 5 with the measured polarization distributions obtained after poling with constant current density. We find the 


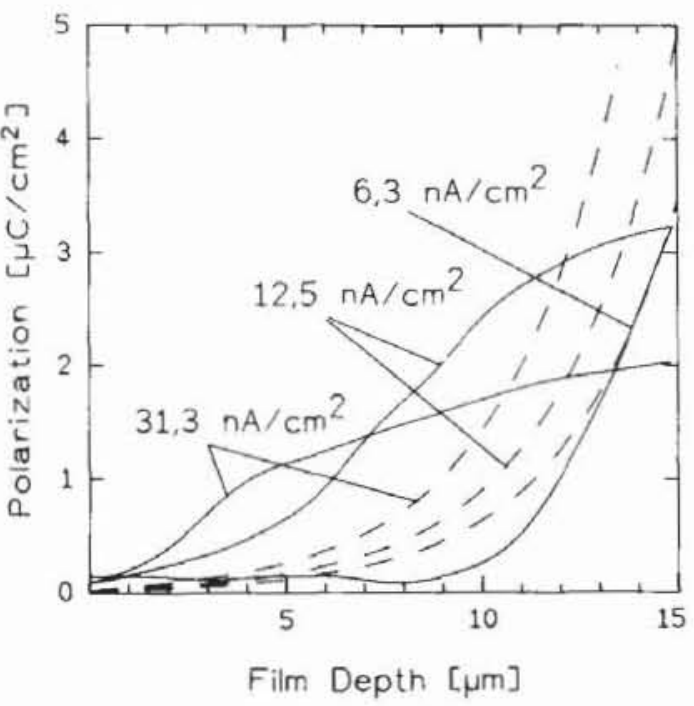

predicted polarization form and the shift of the polarization zone towards the film surface with increasing current density.

We gratefully acknowledge financial support of the Deutsche Forschungsgemeinschaft.

\section{References}

[1] K. Holdik, W. Eisenmenger, in G.M. Sessler (ed.), Proc. 5th Int. IEEE Symp. Electrets, Piscataway, pp. 553-558, 1985.

[2] M. Haardt, W. Eisenmenger, IEEE 1982 Annual Report Conf. Elect. Insul. and Dielect. Phen., 46-51, Piscataway 1982.

[3] W. Eisenmenger, M. Haardt, Solid State Comm., Vol.41, 917-920, 1982.

[4] W. Eisenmenger, M. Haardt, K. Holdik, IEEE 1982 Annual Report Conf. Elect. Insul. and Dielect. Phen., 52-57, Piscataway 1982.

[5] W.H.Press, B.P.Flannery, S.A.Teukolsky, W.T.Vetterling, "Numerical Recipes", Cambridge 1988 